\title{
Thinking about persuasive technology from the strategic business perspective: A call for research on cost-based competitive advantage
}

\author{
Xiuyan Shao $(-)$ [0000-0001-6550-9025] and Harri Oinas-Kukkonen \\ Oulu Advanced Research on Service and Information Systems, \\ University of Oulu, P.O. Box 3000, 90014 Oulu, Finland \\ \{xiuyan.shao, harri.oinas-kukkonen\}@oulu.fi
}

\begin{abstract}
Persuasive system features have been extensively examined, and many of them have been shown to be effective in supporting individuals' achieving their behavioral goals and enhancing system use. Also, companies and organizations have utilized persuasive features in their implementations successfully. However, in order to obtain competitive advantage, organizations need to not only take using persuasive features as differentiation strategy, but also to think about cost of developing persuasive systems. While the research on evaluating persuasive features is important, we argue that previous research has ignored the cost of building persuasive features. As a first step in remedying this gap in research, we present and discuss four research directions for studying cost of developing persuasive systems. This study contributes to persuasive technology field by paving the way for a new research area with highly practical implications.
\end{abstract}

Keywords: Persuasive technology; business strategy; cost-based competitive advantage

\section{Introduction}

In recent years, technology has been used increasingly to persuade people and motivate them toward various individually and collectively beneficial behaviors in various domains such as healthcare, sustainability, education, and marketing. As a research domain, persuasive technology encompasses disciplines such as social psychology, communication studies, computer science, and information systems [1].

Fogg [2] defines persuasive technology as "interactive computing systems designed to change people's attitudes and behaviors" (p. 1), and Oinas-Kukkonen and Harjumaa [3] define persuasive systems as information systems designed to "reinforce, change or shape attitudes or behaviors or both without using coercion or deception" (p. 486). Using such systems is based on voluntariness [1] [4]; for a system to be effective, it would have to have enough persuasive power.

In an industry context, persuasive technologies play a key role in providing an effective means to employ large scale, personalized interventions [2]. Existing healthrelated persuasive technologies in commercial form, such as services, or product- 
service combinations, make an attempt at influencing people to adopt a healthier lifestyles. The majority of these services use implementations of influence strategies and other theories from motivation and persuasion research to gain compliance and/or change attitudes or behaviors [cf., 1]. Kelders et al. [5] reviewed the literature on webbased health interventions and finds that the differences in technology and interaction predict adherence to a web-based intervention. In their study, increased interaction with a counselor, and more extensive employment of dialogue support significantly predicted better adherence. Likewise, in automotive domain, Wilfinger et al. [6] suggested to widen the scope of automotive persuasive interfaces to persuade drivers to drive safer or in a more sustainable way. Persuasive technology also shows its importance in sales and marketing. Basten et al. [7], through a virtual supermarket simulation, found that triggers co-located with the target product lead to higher sales of that product.

In academic settings, much of the focus in studying persuasive technology has been comparing the effectiveness of technology with and without persuasive function on changing people's behavior. For example, Shamekhi et al. [8] indicated that patients with chronic stress who have medical group visits with a computer-animated conversational agent have more positive stress management behaviors as compared to patients who have usual care (attending regular meetings with their primary care physician). Some of the previous research has focused on explaining a system's success factors. For instance, Karppinen et al. [4] studied user experiences of a web-based health Behavior Change Support Systems (BCSSs) designed as lifestyle intervention targeting obesity, and suggested that self-monitoring, reminders, tunneling, and social support were those system features that especially helped users to achieve their health behavior change goals. Some of the previous studies have focused the usage of individual persuasive features in different application domains. For example, Lehto and Oinas-Kukkonen [9] studied multiple health-related behavior change support systems targeting alcohol misuses and weight loss, and found that reduction and self-monitoring were the most common system features to support accomplishing users' primary task in these domains. Some of the previous studies have addressed the effectiveness of particular persuasive features, and in several cases the results have been promising whereas some of the results have been less clear. For instance, the role of reminders in the context of interventions for weight loss, physical activity, and promoting a healthier lifestyle have been studied. Schneider et al. [10] suggested that email prompts are quite effective in boosting revisits to the program and Fry and Neff [11] reported that together with personal contact with a counselor the periodic reminders increase the effectiveness of the interventions, whereas Griffiths and Christensen [12] reported that while using an information system reduces depressive symptoms weekly reminders makes no difference in its effectiveness.

Previous research on persuasive technology has highlighted the importance of designing persuasion into technologies. However, the selection criterion of particular persuasive features for the systems under development is not limited to their effectiveness on users' behavior change. To obtain competitive business advantage in industry, system development organizations usually have the pressure of developing and implementing the systems within limited time and budget. Industry environment is argued to be important to key constituents involved with information systems and technologies [13]. 
Industry can influence information technology (IT) artifact, because the design and functionality of most IT applications reflect an industry's core technologies. Besides, market structure influences the features and structure of IT artifacts, for example, when dominant suppliers or customer drive industry standards for electronic data interchange (EDI) and inter-organizational infrastructure. More importantly, system development organizations have to take the industry environment better into consideration to obtain competitive advantage.

In this viewpoint paper, we argue it is time for the field of persuasive technology to more actively think of research from the business strategic perspective. In this paper, we will discuss, under the framework of Persuasive Systems Design (PSD) model [3], how to study the acquisition of competitive advantage by organizations.

\section{Conceptual background: Competitive advantage}

Understanding the sources of sustained competitive advantage has become a major area of study in strategic management. Porter's seminal work does not only address the sources of sustained competitive advantage [14], but also addresses technology, information and competitive advantage in information systems [15]. Porter's generic strategies (see Figure 1) are the most referred approaches upon the strategies for achieving competitive advantage.

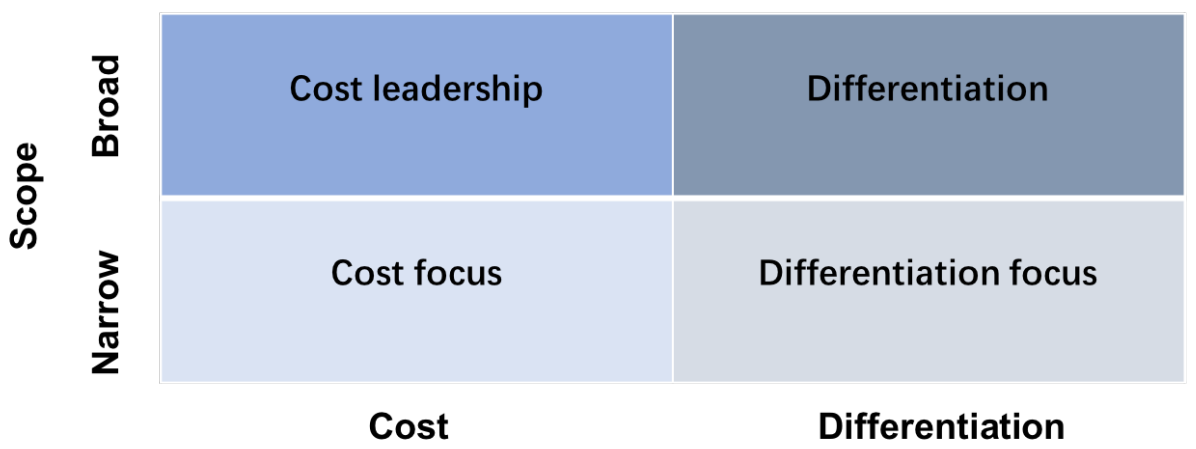

Source of Competitive Advantage

Fig. 1. Porter's Generic Strategies [16]

According to Porter [14], the key of competitive advantage is the ability to set the position of the business against the competition in the market. Companies achieve competitive advantage either by having the lowest product cost or by having products which differ in ways that are customers value. Porter [16] defined these strategic choices namely as cost leadership strategy and differentiation strategy. 


\subsection{Cost leadership}

Cost leadership strategy is expressed as cost advantage reflecting selling the goods and services at a lower cost than competitors in terms of design and production [17]. Cost leadership strategy aims to gain competitive advantage by reducing the costs of R\&D, service, sales and marketing activities [18]. Companies can get competitive advantage in scale of economics by using effective systems to reduce the cost of human resources and minimizing the costs with cheaper raw material, mass production and distribution [19]. Cost leadership provides competitive advantage in the markets in which the consumers are sensitive to the prices. Firms conducting this strategy aim to reduce all cost in the value chain [20].

\subsection{Differentiation}

Second generic business strategy is differentiation. In this strategy, an organization aims to be unique in its sector with some characteristics valued by most buyers. It chooses one or more specific characteristics and it positions itself to meet these needs. In the situations where standard goods and services do not meet customer needs, organizations have to find different solutions to their customers' specific needs. This is another way to compete in the market. The basic principle of the differentiation strategy is channeling the customer choices to its goods and services by doing different things from what every rival is doing [21]. Approaches for differentiation can be composed of elements such as brand name, technology, customer services, sales network and other dimensions. The ideal approach is organization's differentiating itself in several dimensions [15].

In this line of research, specific attention has been given to "competitive advantage" from the main elements of which can be labelled as "product-based" and "cost-based". A significant relationship between product-based advantage and the performance of organizations has been identified. Firms that experience a product-based competitive advantage over their rivals - for example, higher product quality, packaging, design and style - have been shown to achieve relatively better performance [23] [23]. Similarly, research has further illustrated that there is a significant relationship between cost-based advantage and organizational performance. Firms that enjoy cost-based competitive advantages over their rivals - for example, lower manufacturing or production costs, lower cost of goods sold, and lower-price products - have been shown to exhibit comparatively better performance [23] [23].

\section{Competitive advantage in persuasive technology business}

To think about persuasive technology from competitive advantage perspective, Fig. 2 summarizes some of the relevant topics. To differentiate, research has already been conducted to evaluate effectiveness of persuasive features, while future research can consider strategically providing persuasive features to customers. To reduce cost of persuasive technology, research can be conducted to investigate cost related with persuasive features, persuasive system development, and strategic cost analysis. 


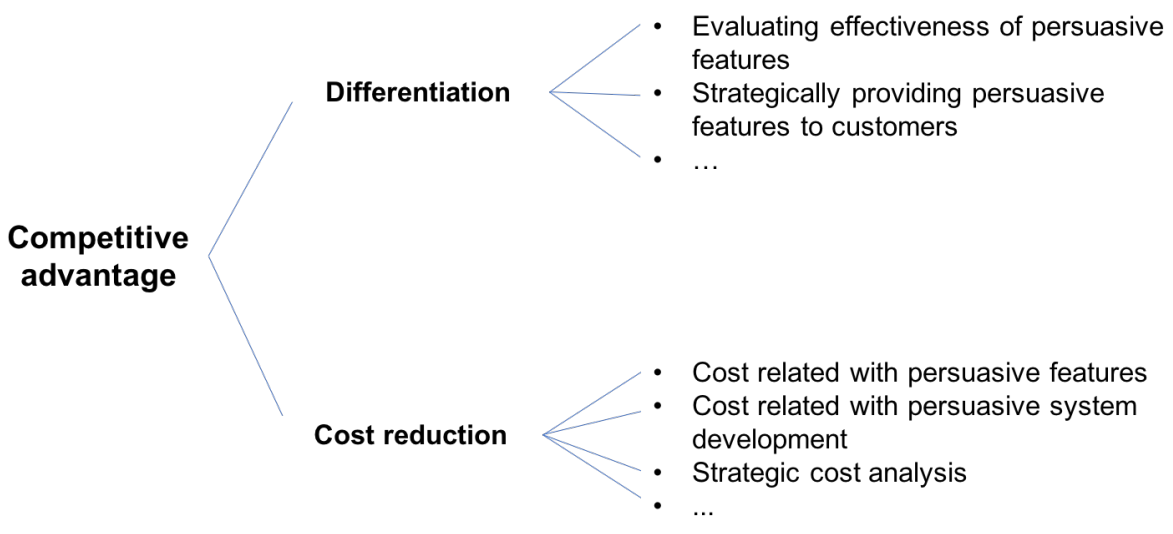

Fig. 2. Competitive advantage in persuasive technology business

Persuasive technology business is still at an immature stage and in many cases a mere provision of persuasive features gives product-based competitive advantage. In health BCSSs, mobile applications that incorporate numerous persuasive features that are being used to modify the behavior of users are being developed [24]. These applications are continually improving and are being used more and more by people in their daily lives. Once more evidence becomes available on the costs of producing the product- or service-based advantage, the persuasive technology as a field has reached a much more mature stage. Yet, research into persuasive systems' cost-based competitive advantage is still almost non-existent. In this section, we discuss in detail how present persuasive technology research has considered competitive advantage. We first discuss differentiation as the strategy under the framework of PSD model. After that, we suggest how to investigate cost-based competitive advantage in persuasive technology research.

\subsection{Differentiation as the strategy}

The differentiation strategy is to create a product or a service, which will differ from the offers by the competitors. In an industry context, designing for persuasion is differentiated from technologies without persuasion or offering a unique persuasive solution. Therefore, this strategy is reflected in persuasive technology research by providing persuasive features and persuasive feature categories that differentiate from competitors.

PSD model. The PSD model [3] is a leading framework in the persuasive systems' field, for designing and evaluating persuasive systems. The PSD model builds on multiple theoretical constructs, such as goal-setting theory [25], the elaboration likelihood model [26], and the theory of reasoned action/planned behavior [27], and it builds upon persuasive technology techniques that Fogg [2], and others define. The PSD model includes a set of seven postulates concerning persuasive systems, and describes 28 potential system features for BCSS, which can be subsumed under the four categories of primary task, dialogue, credibility, and social support features. The PSD model can be 
used as a tool by designers and intervention developers and as a framework for understanding and interpreting users' needs and how these needs can be implemented. In this sense, the PSD model serves as a suitable framework to analyze how persuasive technologies can be different from each other.

The persuasive features in primary task support category aim to reflect individuals' behavior goals and track progress toward them. The dialogue support category consists of persuasive features that are related to human-computer interaction and user feedback. The credibility support category includes features that help in designing more credible and thus more persuasive systems. Finally, persuasive techniques in the social support category aim to motivate users by leveraging social influence.

\section{Providing persuasive features as differentiation strategy}

Primary task support. Oduor and Oinas-Kukkonen [28] found that primary task support has the strongest effect on users' continuance intention of using BCSSs among other persuasive feature categories. In health BCSSs, for example, self-monitoring supports a user's primary task and it has been proven to be an effective persuasive feature [4] [9]. It is also the most common persuasive feature implemented in mobile education applications that promote physical activity [24]. In e-commerce, it has been found that primary task support, together with social support, are strong predictors of perceived effectiveness of an e-commerce company like Amazon [30].

Dialogue support. In healthcare, dialogue support features, together with social support features are also frequently implemented. In terms of computer-human dialogue support, applications utilized user data that had been collected to persuade users to engage in their target behavior. A combination of praise, rewards, reminders and suggestion were used to motivate users to achieve their goals [24]. In e-commerce, dialogue support features significantly influence perceived product credibility and perceived review credibility, which both strongly predict system credibility [30].

System credibility support. Twersky and Davis [29] investigated 32 persuasive technology applications, and found that system credibility support features are the most common type of features. In their findings [29], system credibility support features are found mainly in the applications' websites in their use of supporting platforms (such as Twitter or Google Chrome). However, in other industries, like mobile health education [24], credibility support features are the least mentioned. This is confirmed by Adaji and Vassileva [30], which show that in e-commerce perceived system credibility does not influence continuance intention of shoppers in Amazon.

Social support. To explore how social influence design principles affects customer engagement in sharing feedback, Stibe and Oinas-Kukkonen [31] implemented an information system consisting of social influence design principles, and found that social influence could predict even up to $40 \%$ of the variance in behavioral intention. For instance, in a persuasive strategy design by Wunsch et al. [32], bikers who received social comparison messages increased their biking compared with control group. They suggested that competition and collective goal elements should be designed to allow social comparison also with familiar besides unknown participants.

Persuasive feature categories are usually implemented dependent on each other to achieve higher effectiveness on persuasion. For example, in health BCSSs primary task 
support features such as tailoring and monitoring are often present together with dialogue support features such as reminders, rewards and praise [24]. Applications that included self-monitoring features generally included also a feedback mechanism that allowed the users to review their data.

The PSD model suggests that persuasive features have potential to help persuasive technology be effective. Research has already put an effort into investigating how persuasive features make technology more effective in changing people's behavior. To gain some product-based competitive advantage, organizations can simply implement the proven persuasive features into technologies as the first step. Yet, to achieve greater product-based competitive advantage, market analysis of target customer is needed to understand the unique requirements of the group of customers and provide higher quality persuasive technology.

\subsection{Cost as the strategy}

To achieve cost-based advantage organizations have to have lower costs than their competitors. Finding the way to lower the cost of production may be the most important question for organizations developing persuasive technology. This urges on estimating the costs of developing persuasive systems and the costs of developing persuasive features into them. But this strategic approach is still quite neglected in persuasive technology research. Here we outline four research directions based on cost-based competitive advantage and PSD model to investigate how to take cost-based competitive advantage into account in persuasive technology research.

Research direction 1: Understanding the cost associated with persuasive technology development. To estimate cost related with persuasive system implementation, software cost estimation models can serve as a foundation. Software cost estimation models attempt to generate an effort estimate, which can be converted into the project duration and cost. Software cost estimation models range from empirical models such as Boehm's COCOMO model [33] to analytical models such as those in Putnam [34], Parr [35], and Cantone et al. [36]. Most software cost models are based on the size measure, such as lines of code (LOC) and function points (FP) within persuasive technology. Software cost estimation models can be applied to understand the cost of developing persuasive features. However, to understand all the relevant costs, it is important to first understand the process of persuasive systems development. Interviews can be done with developers to explore more relevant costs. When conducting interviews, three steps suggested by PSD model for persuasive system development [3] can be used as general framework to design interview questions: (i) analysis of persuasion context and selection of persuasive design principles; (ii) requirement definition for software qualities; (iii) software implementation.

A notable issue related to persuasive technology development is that some persuasive features are considered as popular features [29], and some are usually implemented hand in hand. To evaluate the cost of having multiple persuasive features, simply adding the costs of developing individual persuasive features may not be a correct approach. Cost synergy can be studied in the context of implementing multiple persuasive features from many perspectives. For example, economy of scale explains cost 
reduction due to the scale of operation [37]. Often there is an optimum design point where costs per additional unit begin to increase. In the context of implementing persuasive features, future research can explore what is the optimum design point for adding more persuasive features. Future studies can also apply other explanations for cost synergy related with persuasive feature implementation.

Research direction 2: Strategic cost analysis for persuasive system development. To stay cost competitive, strategic cost analysis should be considered. Shifts in cost drivers can be identified. Strategic cost drivers include structural cost driver and executional cost driver. Structural cost drivers usually are affected by organizations' external environment, while executional cost drivers are usually affected by the execution of the business activities such as capacity utilization, plant layout, and work-force involvement [38].

To analyze structural cost drivers, it is important to understand customer needs and maintain customer relationship. Customer's Resource Life Cycle [39], for example, can be applied by future research to identify and categorize strategic orientations by focusing on the possible differentiation of organization's product from competitors' products on the basis of customer service. This model considers an organization's relationship with its customers and how this relationship can be changed or enhanced by the strategic application of information system technologies. The four- and thirteen-stage resource life cycles can serve as framework for future case studies.

Interviews conducted in research direction 1 may help understand what are executional cost drivers in the context of persuasive technology development. To manage executional cost drivers, future research may consider factors such as structure and culture of the organization, total quality management, among other issues.

Research direction 3: Empirically validating the price strategy. A third direction of research could adopt a theory-testing research setting and investigate the effectiveness of price strategy. Theories from information systems, marketing, management, and economics can be applied to form a comprehensive analysis framework. The success of price strategy needs to be defined, for instance, based on the goal of persuasive system development. Success elements may include users' actual use of persuasive technology, users' behavioral change, users' satisfaction with the persuasive technology, market share increase. Research in this direction can be quantitative. For instance, a relevant topic concerns about providing different persuasive features and accordingly pricing to different user groups in order to capture a larger portion of the total market surplus. Most persuasive technologies provide the same functions to all customers despite their heterogeneity in willingness to use. While providing persuasive function costs, it is wise to provide a customer the functions that he/she is mostly willing to use. Group pricing (or third-degree price discrimination) refers dividing the market into segments and charges different price to each segment [40], and can be found in many industries today. The travel, hospitality, and entertainment industries commonly offer special corporate or loyalty discount rates; insurance companies use to classify risks and discriminate fees based on the insuree's age, sex, marital status, occupation, etc. Future research may consider taking experiments in user groups provided with different sets of persuasive features and prices to test their use. 
Research direction 4: Theory development. While the third stream of research called for theory testing, we also see that inductive and qualitative approaches are needed. The limitation of the theory-testing setting is that it merely tests if existing theory is supported or not. In contrast, theory development would approach the problem perhaps even from a clean table without any theories in mind by asking persuasive technology development managers report their considerations about competitive advantage. Ideally, a qualitative approach would allow researchers to develop new constructs, concepts, and even theories that explain the persuasive technology design for competitive advantage. Such in-depth interview studies could also reveal a process that covers several rounds of refinement of persuasive technology development. Possible methods for analyzing the interviews includes grounded theory.

It has to be clarified that the new research directions do not refute the importance of previous research on persuasive systems features evaluation. On the contrary, previous research provides fundamental knowledge for new research directions. Although we emphasized that future research on cost-based competitive advantage is needed, we keep it in mind that the flexibility to have "product-based" and "cost-based" strategy may be necessary for persuasive technology business to gain sustainable competitive advantage.

\section{Discussion}

To gain competitive advantage, a company must either perform these development activities at a lower cost or perform them in a way that leads to differentiation and a premium price [14]. PSD provides a list of persuasive features that offer potential for differentiating persuasive technologies. Numerous studies have investigated effectiveness of these persuasive features on users' behavior change in many application areas. However, so far the research community has paid little attention to the cost of persuasive technology development. The purpose of this viewpoint paper has been to address this oversight and provide research directions for possible future studies. Past research has provided a solid foundation for understanding persuasive features' impact on changing users' behavior, and we now call for new perspectives - especially for analyzing the cost of persuasive features. We argue that to obtain competitive advantage, persuasive technology organizations must not only select from the proven effective persuasive features, but also analyze the cost related with persuasive technology development.

We have framed our arguments through the framework of the PSD model [3], and proposed four research directions for future studies. Although the four proposed research directions have been discussed in isolation, we would suggest that these, and potentially other topics for consideration, might prove fruitful when studied together. For example, research into strategic cost analysis would also appear to link well with the work on understanding costs. As noted, for example, structural cost driver and executional cost driver can be considered in the interviews to understand the cost associated with persuasive technology development. Another area involves consideration of the synergy effect of persuasive features. When multiple persuasive features are 
implemented, do they lead to more significant impact on users' behavior change, and thus be more cost-efficient? One reasonable explanation can be economies of scale, which are the cost advantage that organizations obtain due to their scale of operation, with cost per unit of output decreasing with increasing scale.

This study offers a valuable contribution to research because previous research on persuasive technology mostly examined the relationship between persuasive features and users' behavior change, leaving external and internal business environment currently absent from the persuasive technology literature. A strategic business perspective is therefore important. Future research should take more industry context into consideration. The proposed research directions will provide implications for practice. Understanding cost of persuasive features offers possibility for development organizations to adjust their system development strategy, and achieve cost efficiency and user satisfaction. This is expected to offer more comprehensive perspective for industry.

\section{Conclusion}

Persuasive technology literature has put much focus on examining the impacts of persuasive features on users' behavioral change, and this provides significant insights for technology developers. To design a successful persuasive technology, persuasive features can be selected from what's already proved to be effective. To obtain competitive advantage for development organizations, the selection of persuasive features should also consider organizational financial issues and industry environment. We call for research that takes a competitive advantage attitude, especially the cost-based competitive advantage perspective, into persuasive design. As a first step in remedying the gap in our understanding, this study opens up a new research area in persuasive technology field, and our study highlights several directions for future research. First, understanding the cost associated with persuasive technology development. The steps suggested by PSD model can be taken into account when interviewing organizations regarding with the relevant costs. Software cost estimation models can be learnt from. Second, future research could also explore the management of strategic cost. Organization's external environment such as competition shifts should be taken into account. Third, research that empirically validates the price strategy can be done to show if price strategy really matters. Finally, we suggest that future research to develop theory specifically in persuasive technology field related with product-based competitive advantage and cost-based competitive advantage. We believe that the proposed research directions will contribute to the body of knowledge on persuasive technology research.

\section{References}

1. Oinas-Kukkonen, H.: A foundation for the study of behavior change support systems. Personal and Ubiquitous Computing, 17(6), 1223-1235 (2013)

2. Fogg, B.J.: Persuasive Technology: Using Computers to Change What We Think and Do. Morgan Kaufmann Publishers, Inc., San Francisco (2003). 
3. Oinas-Kukkonen, H., Harjumaa, M.: Persuasive systems design: Key issues, process model, and system features. Communications of the Association for Information Systems, 24, 485500 (2009)

4. Karppinen, P., Oinas-Kukkonen, H., Alahaivala, T., Jokelainen, T., Keranen, A. M., Salonurmi, T., Savolainen, M.: Persuasive User Experiences of a Health Behavior Change Support System: A 12-Month Study for Prevention of Metabolic Syndrome. International Journal of Medical Informatics 96, 51-61 (2016)

5. Kelders, S.M., Kok, R.N., Ossebaard, H.C., Van Gemert-Pijnen, J.E.W.C.: Persuasive system design does matter: a systematic review of adherence to web-Based interventions, J. Med. Internet Res. 14, 17-40 (2012)

6. Wilfinger, D., Gartner, M., Meschtscherjakov, A., Tscheligi, T.: Persuasion in the Car: Probing Potentials. In: Proceedings of Persuasive 2014 (2014)

7. Basten, F., Ham, J., Midden, C., Gamberini, L., Spagnolli, A.: Does Trigger Location Matter? The Influence of Localization and Motivation on the Persuasiveness of Mobile Purchase Recommendations. In: Proceedings of Persuasive 2015 (2015)

8. Shamekhi, A., Bickmore, T., Lestoquoy, A., Gardiner, P.: Augmenting Group Medical Visits with Conversational Agents for Stress Management Behavior Change. In: Proceedings of Persuasive 2017 (2017)

9. Lehto, T., Oinas-Kukkonen, H.: Examining the Persuasive Potential of Web-Based Health Behavior Change Support Systems. Transactions on Human-Computer Interaction, 7(3), 126140 (2015)

10. Schneider, F., de Vries, H., Candel, M., van de Kar, A., van Osch, L.: Periodic email prompts to re-use an Internet-delivered computer-tailored lifestyle program: Influence of prompt content and timing. Journal of Medical Internet Research, 15(1), e23. (2013)

11. Fry, J.P., Neff, R.A.: Periodic prompts and reminders in health promotion and health behavior interventions: A systematic review. Journal of Medical Internet Research, 11(2), e16. (2009)

12. Griffiths, K. M., Christensen, H.: Internet based mental health programs: A powerful tool in the rural medical kit. Australian Journal of Rural Health, 15(2), $81-87$ (2007)

13. Chiasson, M. W., Davidson, E.: Taking industry seriously in information systems research. MIS Quarterly, 29(4): 591-605 (2005)

14. Porter, M. E.: Competitive advantage: Creating and sustaining superior performance. New York: The Free Press. (1985)

15. Porter, M. E., Millar, V. E.: How information gives you competitive advantage. Harvard Business Review, 63(4): 149-160 (1985)

16. Porter, M.E.: New global strategies for competitive advantage. Planning Review, 18(3),4-14 (1990)

17. Wheelen, T. L., Hunger, D. J.: Strategic management and business policy (8th ed.). Massachusetts: Prentice Hall. (2002)

18. Rugman, A., Hodgetts, R.: International business: A strategic management approach (2nd ed.). New York and London: Prentice Hall (2000)

19. Eraslan, I.H.: The effects of competitive strategies on firm performance: A study in Turkish textile and apparel industry considering the mediating role of value chain activities. Bogazii University Social Sciences Institute, Istanbul. (2008)

20. Thompson, A. A., Strickland, A. J.: Strategic management: Concepts and cases. (9th ed.). USA: Irwin McGraw-Hill. (1996)

21. Bal, Y.: The effect of competitive strategies on human resource management practices. Istanbul University Institute of Social Sciences, Istanbul. (2011) 
22. Gimenez, C., Ventura, A.: Supply chain management as a competitive advantage in the Spanish grocery sector. Published Working Paper. No. 2, 04/2002, Universitat Pompeu Fabra' (UPF), Barcelona, Spain. (2002)

23. Morgan, N. A., Kaleka, A., Katsikeas, C. S.: Antecedents of export venture performance: A theoretical model and empirical assessment. Journal of Marketing, 68, 90-108 (2004)

24. Matthews, J., Win, K.T., Oinas-Kukkonen, H., Freeman, M.: Persuasive Technology in Mobile Applications Promoting Physical Activity: A Systematic Review. Journal of Medical Systems 40 (3): 72 (2016)

25. Locke, E. A., Latham, G. P.: Building a practically useful theory of goal setting and task motivation: A 35-year odyssey. American Psychologist, 57(9), 705-717 (2002)

26. Petty, R. E., Cacioppo, J. T.: The elaboration likelihood model of persuasion. Advances in Experimental Social Psychology, 19, 123-205 (1986)

27. Ajzen, I.: The theory of planned behavior. Organizational Behavior and Human Decision Processes, 50(2), 179-211 (1991)

28. Oduor, M., Oinas-Kukkonen, H.: Commitment Devices as Behavior Change Support Systems: A Study of Users' Perceived Competence and Continuance Intention. In: Proceedings of Persuasive 2017 (2017)

29. Twersky, E., Davis, J.: Don't Say That! A Survey of Persuasive Systems in the Wild. In: Proceedings of Persuasive 2017 (2017)

30. Adaji, i., Vassileva, J.: Perceived Effectiveness, Credibility and Continuance Intention in Ecommerce: A Study of Amazon. In: Proceedings of Persuasive 2017 (2017)

31. Stibe, A., Oinas-Kukkonen, H.: Using Social Influence for Motivating Customers to Generate and Share Feedback. In: Proceedings of Persuasive 2014 (2014)

32. Wunsch, M., Stibe, A., Millonig, A., Seer, S., Dai, C., Schechtner, K., Chin, R. C.C.: What Makes You Bike? Exploring Persuasive Strategies to Encourage Low-Energy Mobility. In: Proceedings of Persuasive 2015 (2015)

33. Boehm, B. W.: Software engineering economics. Englewood Cliffs, NJ: Prentice-Hall. (1981)

34. Putnam, L. H.: A general empirical solution to the macro software sizing and estimating problem. IEEE Transactions on Software Engineering, SE-4(4), pp. 345-361, July 1978

35. Parr, N. A.: An alternative to the Raleigh Curve Model for Software development effort. IEEE on Software Eng. May 1980

36. Cantone, G., Cimitile, A., De Carlini, U.: A comparison of models for software cost estimation and management of software projects. Computer Systems: Performance and Simulation, Elisevier Science, Amsterdam. (1986)

37. Panzar, J.C., Willig, R. D.: Economies of scale in multi-output production. Quarterly Journal of Economics, 91, 481-493 (1977)

38. Shank, J. K., Govindarajan, V.: Strategic cost management. New York: The Free Press. (1993)

39. Ives, B and Learmonth G. P.: The information system as a competitive weapon. Communications of the ACM, 27(12): 1193-1201(1984)

40. Varian H. R.: Price discrimination and social welfare. The American Economic Review. 75(4): 870-875 (1985) 